\title{
IMPACT OF VARIOUS FACTORS ON PROBABILITY OF REACHABILITY IN MANET: A SURVEY
}

\author{
Chander Kumar ${ }^{1}$, Shailender Gupta ${ }^{2}$ and Bharat Bhushan ${ }^{3}$ \\ ${ }^{1}$ Department of Computer Engineering, \\ YMCA University of Science and Technology, Faridabad, India \\ nagpalckumar@rediffmail.com \\ ${ }^{2}$ Department of Electronics Engineering, \\ YMCA University of Science and Technology, Faridabad, India \\ shailenderegmail.com \\ ${ }^{3}$ Department of Electronics Engineering, \\ YMCA University of Science and Technology, Faridabad, India \\ bhrts@yahoo.com
}

\begin{abstract}
The Probability of Reachability (POR) is defined as fraction of possible reachable routes to all possible routes between all different sources to all different destinations. In network like Mobile Ad-hoc Network (MANET) adequate level of POR is desirable for its smooth functioning. Its value depends upon various factors such as Transmission Range (T), Number of Nodes (N), node mobility, channel fading, shape and size of the region where the ad-hoc network is to be deployed. To find the impact of N,T, size and shape on the value of POR, a shortest path routing algorithm was implemented in MATLAB and effect of the above said parameters was studied. We observe significant impact of varying not only $N$ and $T$ but also of varying size and shape of the region on the POR values.
\end{abstract}

\section{KEYWORDS}

Ad-hoc network, collision, routing protocol

\section{INTRODUCTION}

Routing is a process to establish a route from source to destination. The design of routing protocol for the ad-hoc network like MANET is a challenging task even today for researchers due to multiple constraints such as limited bandwidth, transmission range, residual battery power and computational capacity of the nodes. In spite of all these constraints it mandatory to have a good connectivity among the nodes of ad-hoc network. It is therefore necessary for routing protocols to provide good POR. Mathematically:

$$
\mathrm{POR}=\frac{\sum \text { Reachable Paths between Source and Destination }}{{ }^{\mathrm{n}} \mathrm{C}_{2}}
$$

where $n$ is no of nodes deployed.

This network parameter is a very important as it tells us about quality of connectedness of the network nodes. If the POR value is very low, it may result into partitioning of the network, defying the very purpose of MANET. On the other hand if POR value is very high it may indicate

DOI : 10.5121/jgraphoc.2011.3302 
International journal on applications of graph theory in wireless ad hoc networks and sensor networks

(GRAPH-HOC) Vol.3, No.3, September 2011

over deployment of resources which may not be a desirable feature in network like MANETs. In order to have adequate value of POR, various factors to be taken into consideration are: transmission range, mobility, multi-path fading, number of nodes deployed and size and shape of the region where the network is to be deployed.

In this paper we try to find the impact of varying number of nodes deployed in MANET, transmission range of nodes, size and shape of the region on the POR values. For this purpose a simulator was designed in MATLAB. The results obtained show a significant impact of these parameters on POR.

The rest of the paper has been organized as follows: In Section 2, we provide the literature survey of this field. In section 3, the design of the simulator and experimental set up is shown. Section 4 provides the simulation results along-with their analysis. Section 5 provides concluding remarks.

\section{LITERATURE SURVEY}

MANET literature provides research on the impact of various parameters on POR values. The literature shows that the POR values depend on the following factors:

Transmission Range: The transmission range is a vital parameter that affects the POR. When it is too high, helps in having high POR value but at the same time results in over consumption of the transmission energy as it is directly proportional to transmission range [2]. On the other hand when transmission range is low, it results in lower value of POR, which is not a desirable feature in MANET [2, 12].

Mobility: As the nodes in MANETs are mobile, its topology is dynamic that results in fluctuations in neighbours per node, therefore the POR value may decrease to unacceptable levels $[3,13]$. It is also shown by many researchers that different mobility models [14] [15] [16] have different impact on POR values.

Multi-path Fading: The atmospheric condition like multi-path fading also affects the POR. Choong Hock MAR [3] studied the impact of multi-path fading on connectivity probability under the Rayleigh fading channel. It is observed that as the values of $\mathrm{N}$ and $\mathrm{T}$ fluctuates under fading, connectivity decreases significantly.

Number of Nodes: The number of nodes deployed in a given region also plays an important role in providing good POR. If the number of nodes is too low then it results in very low POR. On the other hand if it is too high then it results in high POR value but at the same time results in unnecessary wastage of resources [2].

Francois Ingelrest et al. [2] studied the combined impact of $\mathrm{N} \& \mathrm{~T}$ to achieve $100 \%$ POR and gave following relation:

$$
N^{*} T^{2}=\text { constant }
$$

Since the study was done with uniform distribution of nodes in a given region, these results were not applicable to network like MANET where topology is dynamic and the number of neighbours per node keeps on changing. For this scenario various approaches are proposed in $[4,5,6,7,8]$ to achieve $100 \%$ POR. They introduced a term magic number which is defined as the number of neighbours per node to achieve $100 \%$ POR. It was proposed that the magic number should be in the range 5 to $8[9,10,11]$. 
International journal on applications of graph theory in wireless ad hoc networks and sensor networks

(GRAPH-HOC) Vol.3, No.3, September 2011

Shape: The shape of the region in which ad-hoc network is to be deployed have a significant impact on the POR value. N. Meghanathan et. al [1] studied the impact of shape on connectivity and found that as the shape of the ad-hoc network changes from square to rectangle the connectivity is affected i.e. more is the length to breadth ratio $(>1)$ lesser is the connectivity.

The above mentioned research is limited to square or rectangular shaped region of fixed size. Our contribution to the research is to carry out exhaustive study of impact on POR for large ranges of $\mathrm{N}, \mathrm{T}$, size and shape of region.

\section{SIMULATOR DESIGN AND EXPERIMENTAL SETUP}

A shortest path routing protocol was implemented in MATLAB using Dijkstra's algorithm. To calculate the POR value a counter is used which is initialized to zero. The routes from all source nodes to all destination nodes are calculated. If the path exists between a particular source destination pair then the value of the counter is increased by one. At the end of every iteration the POR is calculated. This process is repeated for 25 iterations and average value of POR is taken. The whole process is explained with the help of flowchart as shown in Figure 2. Table 1 shows the basic simulation parameters along-with their values used in simulation. The snapshots of different shapes used in the simulation are also shown in Figures 2a. - 2g. The random distribution of nodes in the region along-with a route between a sample source destination pairs is shown. The experimental set up to record impact of various factors is as follows:

Table 1. Simulation set up parameters

\begin{tabular}{|l|l|l|}
\hline \multicolumn{2}{|l|}{ Parameter } & Value \\
\hline \multicolumn{2}{|l|}{ Size of the region } & 250000 to 2250000 sq. unit \\
\hline \multicolumn{4}{|l|}{ Shape of the } \\
region & Shape 1 & 1. Square $: 1500$ units X 1500 units \\
& Shape 2 & 2. Rectangle :3000 units X 750 units \\
& Shape 4 & 3. Rectangle : $: 1875$ units X 1200 units \\
& Shape 5 & 5. Rectangle :4800 units X 468.75 units \\
& Shape 6 & 6. Rectangle :2400 units X 937.50 units \\
Shape 7 & 7. Ellipse : 1500 units, 477.27 unit (major \\
\hline Number of nodes deployed & minor axis) \\
\hline Transmission range & 20, 40, 60, 80 and 100 \\
\hline Routing algorithms used & 200 to 600 varied in step of 5 \\
\hline Placement of nodes & Dijkstra's shortest path algorithm \\
\hline No. of iterations & Random using Rand() function \\
\hline
\end{tabular}

Set up for transmission range: The shape is chosen one at time from seven different shapes and the number of nodes is varied from 20-100 with step size 20. The size of the region taken is 2250000 sq. units. For all combination of shapes and number of nodes the POR results are recorded by varying transmission range from 200 to 600 in steps of 5 units.

Set up for number of nodes: The shape is chosen one at a time from seven different shapes and the transmission range chosen is 200 to 600 units increasing in step of 100 units. Size of the region is fixed at 2250000 sq units. For all combinations of shapes and the transmission ranges the POR results are recorded by varying number of nodes from 20 to 100 in steps of 5 .

Set up for shape: The number of nodes are varied from 20-100 with step size of 20 in the region of fixed size of $2250000 \mathrm{sq}$ units. The transmission range of the node is fixed at 200 units. The POR results are calculated for seven different shapes. 
Set up for size: To study the impact of size on POR a square shaped region is chosen whose size is changed from 250000 to 2250000 sq. units in step of 250000 sq. units. For each choice of size the number of nodes deployed in region is 20 and transmission range of the nodes is fixed at 200 units.

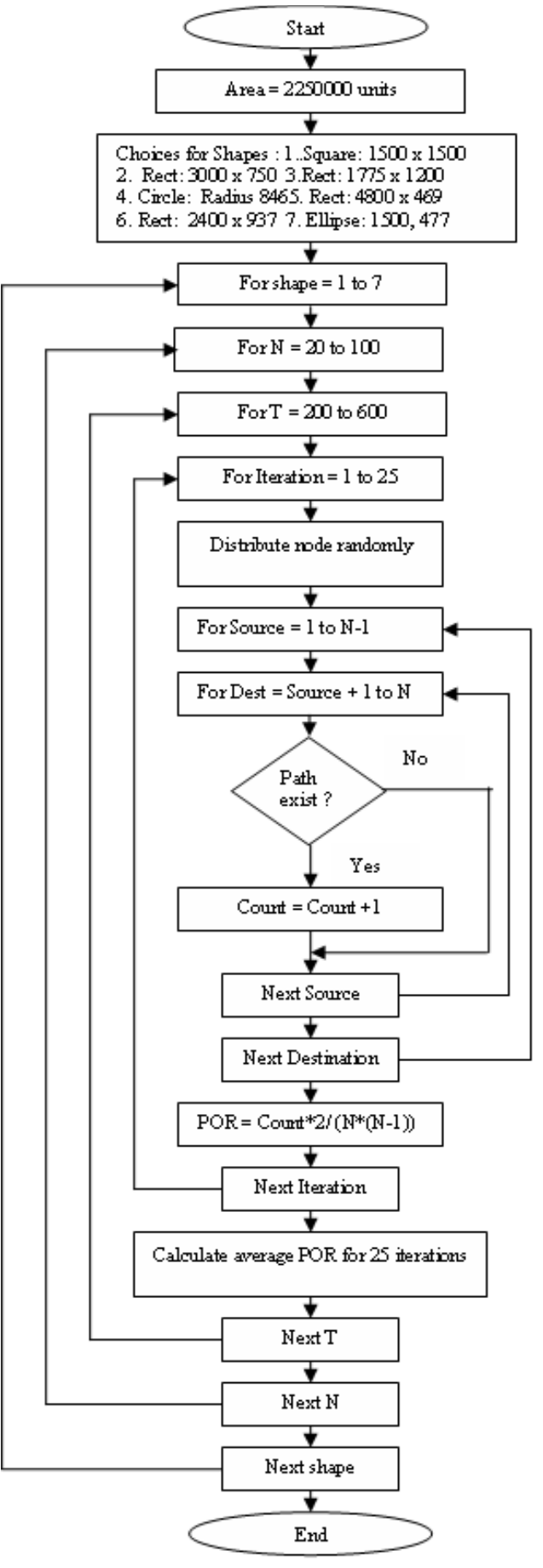

Figure 1. Simulator flow chart

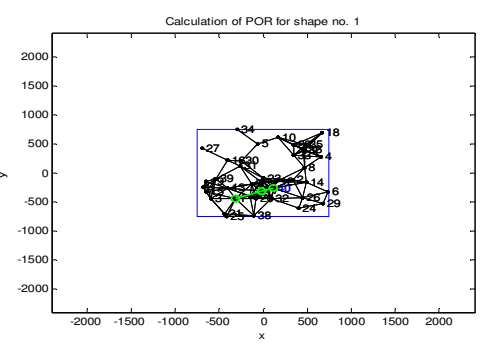

Figure 2a. Snapshot for shape 1

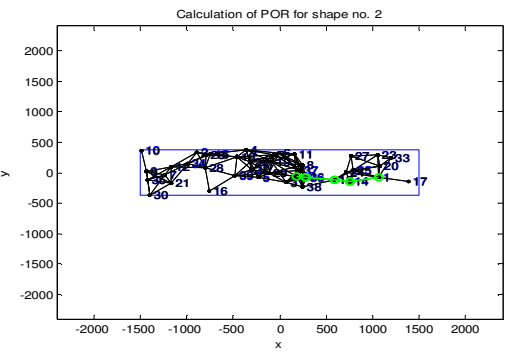

Figure 2b. Snapshot for shape 2

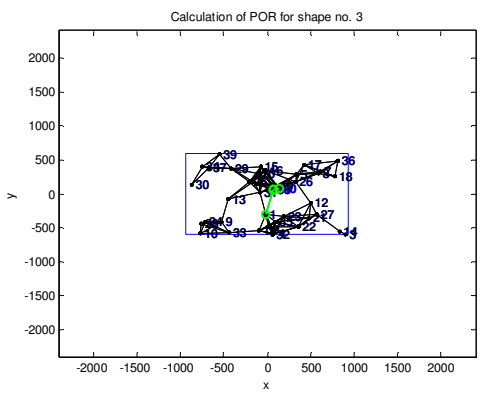

Figure 2c. Snapshot for shape 3

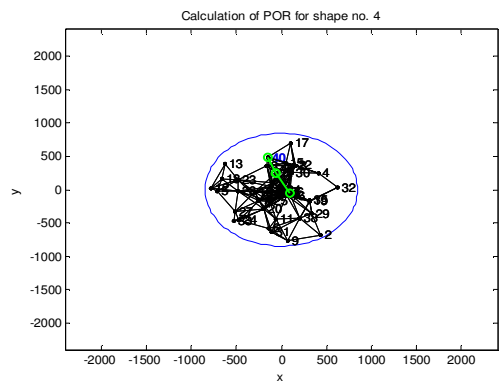

Figure 2d. Snapshot for shape 4 
International journal on applications of graph theory in wireless ad hoc networks and sensor networks

(GRAPH-HOC) Vol.3, No.3, September 2011
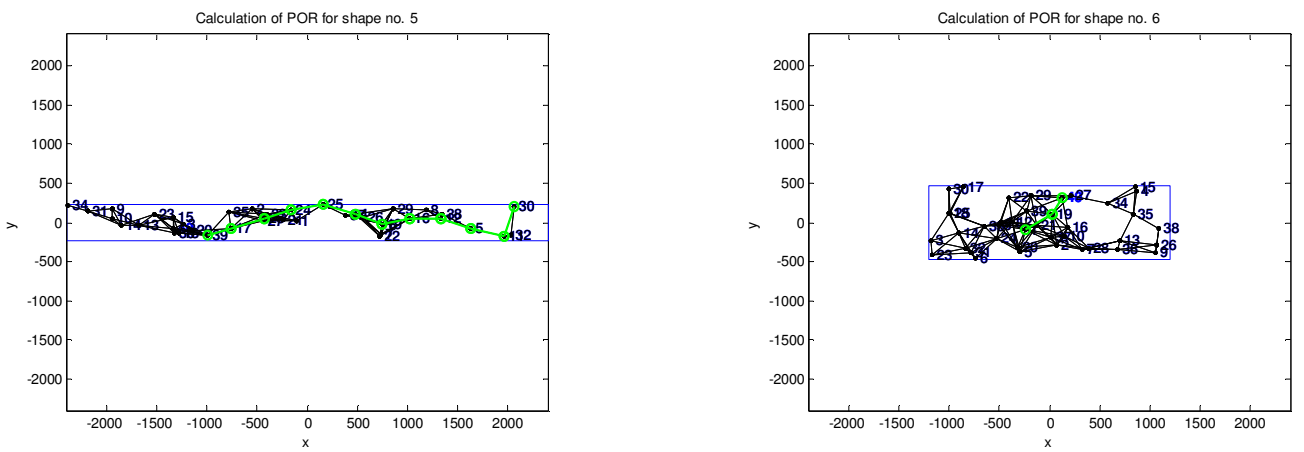

Figure 2e. Snapshot for shape 5

Figure 2f. Snapshot for shape 6

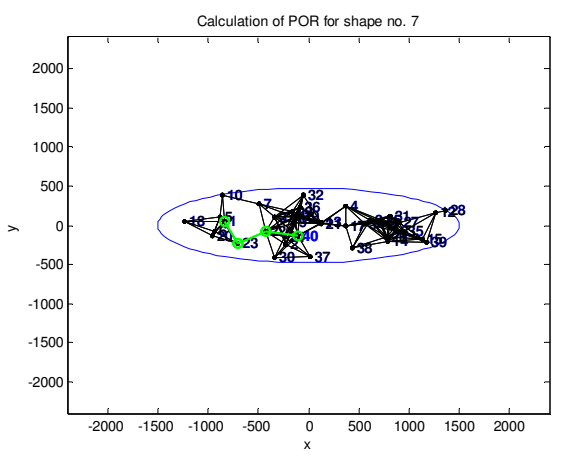

Figure 2g. Snapshot for shape 7

\section{RESULTS AND DISCUSSION}

The impact of various parameters on POR is described as follows:

\subsection{Impact of transmission range}

Figures 3a. to 3g. show the impact of varying transmission range on POR values for seven different shapes. The following inferences can be drawn from the results:

- As the transmission range of the node increases, the area covered by a node increases which results in increase of the neighbours per node that ultimately leads to high POR values.

- As the POR approaches unity the slope of the curves tends to be zero i.e. very high increment in $\mathrm{T}$ is required for marginal increase in POR values. High value of transmission range implies unnecessary wastage of battery power. The POR value chosen should be a trade off between network connectedness and minimum consumption of battery power.

- As the number of nodes increases, the POR increases more rapidly for each and every shape where ad-hoc network is to be deployed. It implies that we can use smaller transmission range to achieve same POR by employing higher number of nodes and vice versa. 
International journal on applications of graph theory in wireless ad hoc networks and sensor networks (GRAPH-HOC) Vol.3, No.3, September 2011

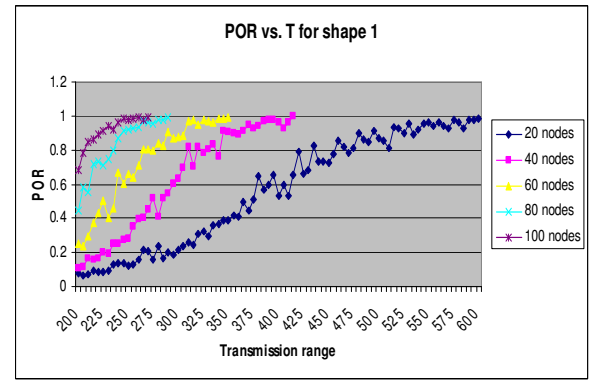

Figure 3a. POR vs. T for 20 to 100 nodes for shape 1

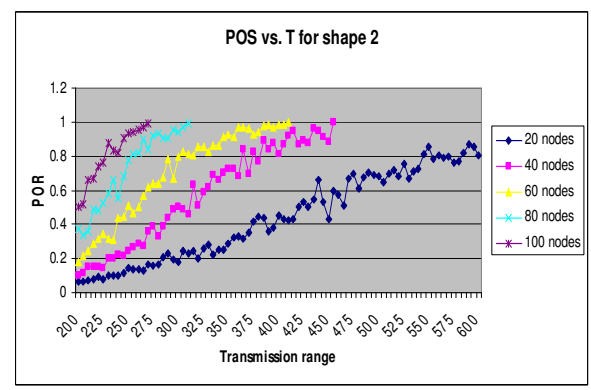

Figure $3 \mathrm{~b}$. POR vs. T for 20 to 100 nodes for shape 2

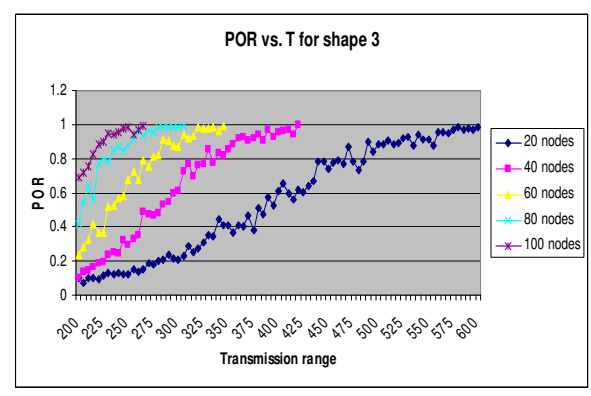

Figure 3c. POR vs. T for 20 to 100 nodes for shape 3

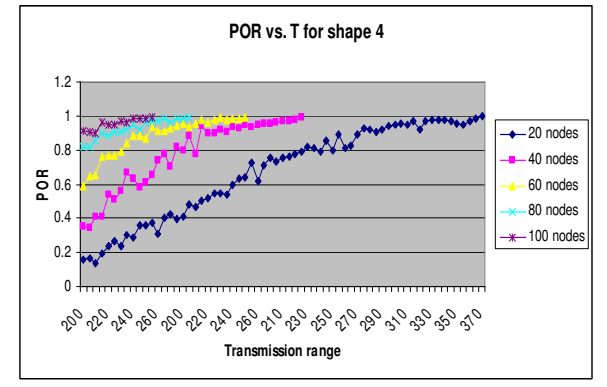

Figure 3d. POR vs. T for 20 to 100 nodes for shape 4

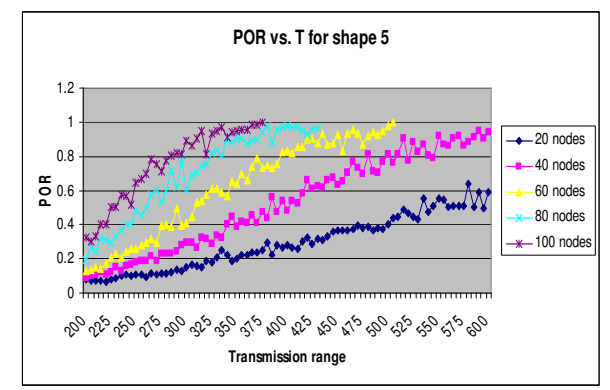

Figure 3e. POR vs. T for 20 to 100 nodes for shape 5

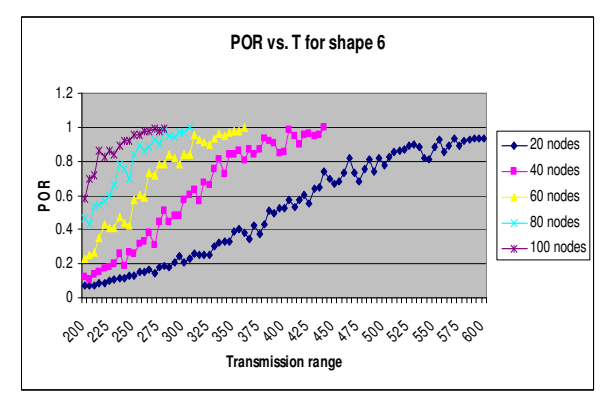

Figure 3f. POR vs. T for 20 to 100 nodes for shape 6

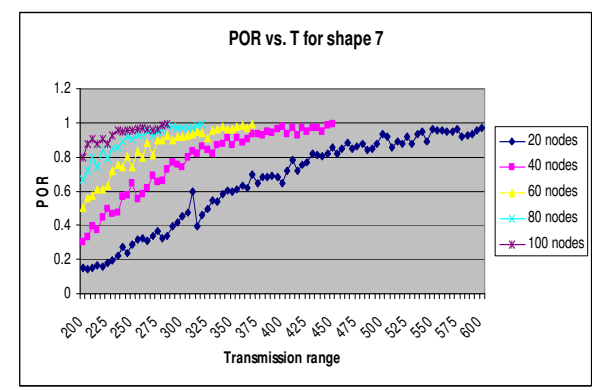

Figure 3g. POR vs. T for 20 to 100 nodes for shape 7 
International journal on applications of graph theory in wireless ad hoc networks and sensor networks

(GRAPH-HOC) Vol.3, No.3, September 2011

- For circular shape the POR value is highest among all given shapes for a given transmission range and number of nodes. This is due to periphery effect which is "As the periphery of the region increases the number of nodes lying on the periphery increases which have half the number of neighbour per node compared to number of neighbours for nodes placed away from periphery." Since the periphery of the circular shape is smallest among all shapes for a given size, the number of neighbours per node is highest; as a result the POR value is highest. Also it may be observed that for the circular shape the POR value reaches unity faster than any other shape.

- On comparison of the result of circular shape and elliptical shape we observe that the ellipse has higher periphery and hence higher periphery effect and lower POR. On comparison of the result of square shape and rectangular shape 3 , we observe that the POR value is lower for rectangular shape due to periphery effect. As we increase length to breadth ratio of the region, the periphery effect goes on increasing and POR value goes on decreasing. The descending order of shapes on the basis of POR values is as follows:

$$
\begin{array}{ll}
\text { 1. } \text { Circle } & \text { : Radius }=846.11 \text { units } \\
\text { 2. Ellipse } & : \text { Major Axis }=1500 \text { units, Minor Axis }=477.27 \text { units } \\
\text { 3. Square } & : \text { Side }=1500 \text { units } \\
\text { 4. Rectangle } & : \text { Length }=1875 \text { units, Breadth }=1200 \text { units } \\
\text { 5. Rectangle } & : \text { Length }=2400 \text { units, Breadth }=937.50 \text { units } \\
\text { 6. Rectangle } & : \text { Length }=3000 \text { units, Breadth }=750 \text { units } \\
\text { 7. Rectangle } & : \text { Length }=4800 \text { units, Breadth }=468.75 \text { units }
\end{array}
$$

\subsection{Impact of number of nodes}

Figures 4a. to 4g. show the impact of varying number of nodes on POR for seven different shapes. The following inferences can be drawn from the results:

- As the number of nodes increases, number of neighbours per node increases thus the POR value increases for all of the shapes.

- For all the shape, as the POR approaches unity the slope of the curves tends to be zero i.e. very high increments in $\mathrm{N}$ is required for marginal increase in POR. Large value of $\mathrm{N}$ is very costly in terms of resources requirements. Hence a trade-off is required between adequate POR value and allocation of resources.

- For circular shape the POR value is highest among all shapes for a given value of $\mathrm{N}$. The reason for the same has been discussed in the previous subsection.

- For circular shape the POR value reaches unity faster than any other shape. Again it is attributed to periphery effect, since the circle has smallest periphery and has smallest periphery effect as a result of which it has highest value of POR.

- As the transmission range is increased, the POR increases more rapidly for each and every shape of the region where ad-hoc network is to be deployed. The increase of transmission range increases no. of neighbours per node, which results in increase of POR. It implies that we can use lesser no. of nodes if we employ higher transmission range to achieve same POR. 
International journal on applications of graph theory in wireless ad hoc networks and sensor networks (GRAPH-HOC) Vol.3, No.3, September 2011

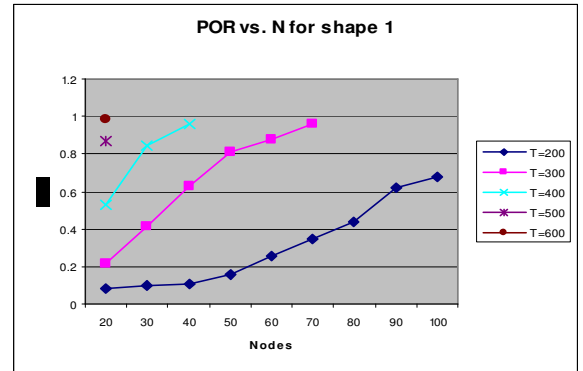

Figure 4a. POR vs. $\mathrm{N}$ for $\mathrm{T}=200$ to 600 unit for shape 1

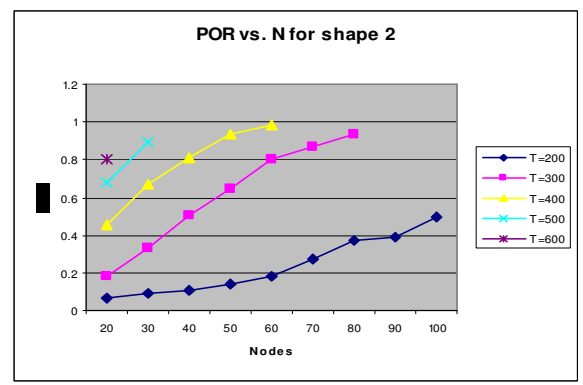

Figure 4b. POR vs. $\mathrm{N}$ for $\mathrm{T}=200$ to 600 unit for shape 2

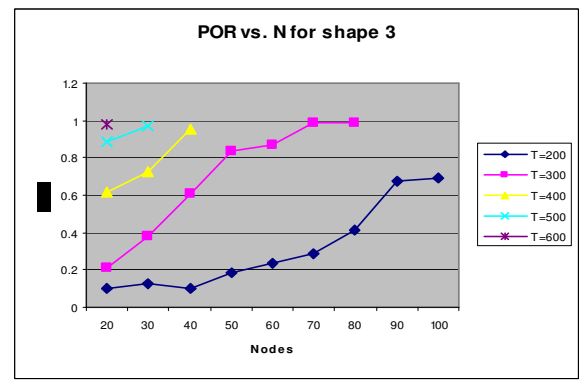

Figure 4c. POR vs. $\mathrm{N}$ for $\mathrm{T}=200$ to 600 unit for shape 3

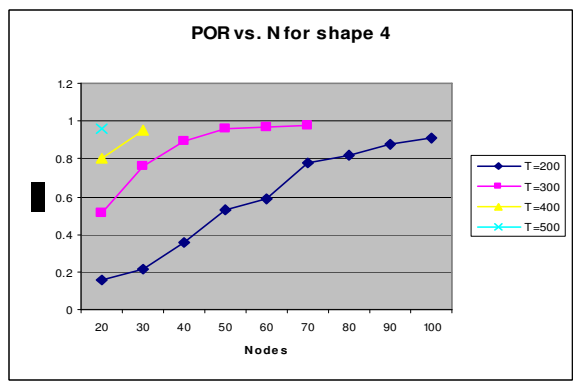

Figure 4d. POR vs. $\mathrm{N}$ for $\mathrm{T}=200$ to 600 unit for shape 4

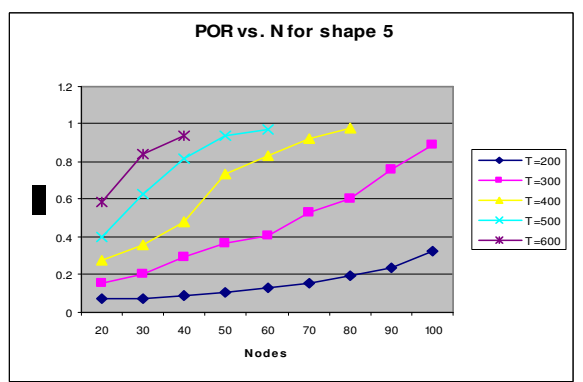

Figure 4e. POR vs. $\mathrm{N}$ for $\mathrm{T}=200$ to 600 unit for shape 5

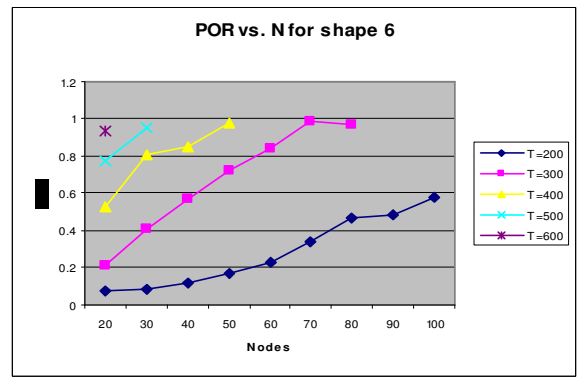

Figure 4f. POR vs. $\mathrm{N}$ for $\mathrm{T}=200$ to 600 unit for shape 6

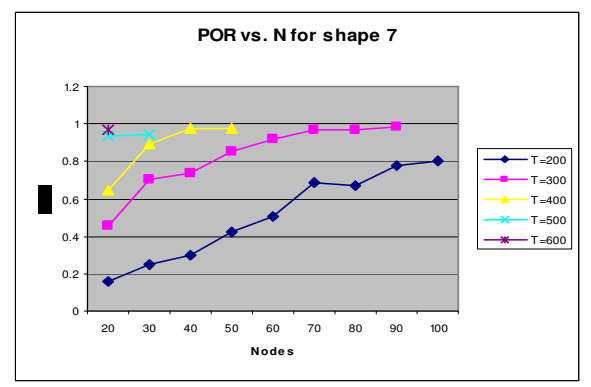

Figure 4g. POR vs. $\mathrm{N}$ for $\mathrm{T}=200$ to 600 unit for shape 7 


\subsection{Impact of size}

Figure 5 shows the impact of varying size on POR for square shaped region having 20 numbers of nodes with each node having fixed transmission range of 200 units. The following inferences can be drawn from the result:

- As the size of the region increases, the node density decreases, number of neighbours per node decreases which ultimately leads to decrease in POR.

- The POR starts from unity and at approaches zero within size interval of 250000 to 2250000 sq. units for fixed of $\mathrm{T}, \mathrm{N}$ and shape.

- Though the results are shown for square shaped region only, similar results can be obtained for other shapes and therefore same remarks are applicable.

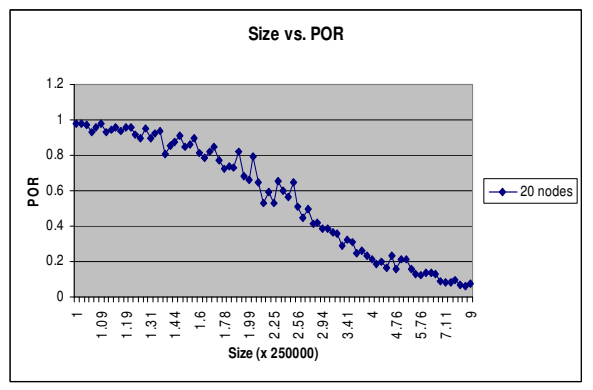

Figure 5. POR vs. Size for $\mathrm{T}=200$ and $\mathrm{N}=20$ for shape 1

\subsection{Impact of shape}

Figure 6 shows the impact of varying shape of the region on POR with fixed transmission range of 200 units and for different value of number of nodes (20-100 with step size $=20)$. The following inferences can be drawn from the results:

- The POR is highest for circular shape for all value of number of nodes. In circular shape the no. of neighbors per node is highest due to periphery effect resulting into highest value of POR.

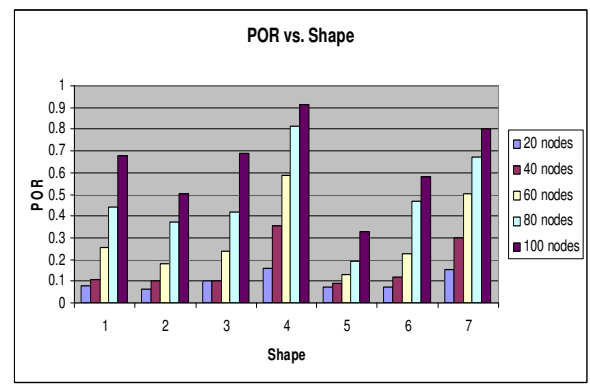

Figure 6. POR vs. Shape for $\mathrm{T}=200$ and $\mathrm{N}=20,40,60,80$ and 100 , size $=2250000$

- In case of square and rectangle, it is seen that as the ratio of length to breadth for a region increases, the periphery effect increases, which result into decrease of POR. 
International journal on applications of graph theory in wireless ad hoc networks and sensor networks

(GRAPH-HOC) Vol.3, No.3, September 2011

- For a particular shape, the POR value increases steadily with increase in N. However marginal increase in POR is smaller for higher value of $\mathrm{N}$.

- Though the results have been shown for $\mathrm{T}=200$ and size $=2250000$ sq. unit, similar results can be obtained for other values of $\mathrm{T}$ and size.

\section{Conclusions}

Following conclusions may be drawn from the results:

- For given values of $\mathrm{N}$ and $\mathrm{T}$ the POR value is highest for circular shape as compared to any other shape. In other words to have same POR value the circular shape requires smallest value of $\mathrm{N}$ and $\mathrm{T}$ hence resource requirements are minimum for circular shape.

- The POR consistently increases with increase in transmission range and/or number of nodes. However marginal increase in POR is low for large increase in $\mathrm{N}$ or $\mathrm{T}$ values when POR is near to unity. Hence achieving unity POR may be exorbitantly costly.

- The values of $\mathrm{N}$ and $\mathrm{T}$ have inverse relation to achieve a given POR value i.e. value of $\mathrm{T}$ should be decreased if value of $\mathrm{N}$ is increased and vice versa. Use of this inverse relation can be helpful in optimising the resources requirements.

- Increase in the size of region results in scarceness of the nodes in a region resulting in fall of POR value.

- The resource requirements are dependent on periphery effect which increases with the increase in periphery of the region. The periphery effect and hence resource requirement for an ad-hoc network can be arranged in following order.

$\begin{array}{lll}\text { 1. } & \text { Circle } & \text { : Radius }=846.11 \text { units } \\ \text { 2. } & \text { Ellipse } & : \text { Major Axis }=1500 \text { units, Minor Axis }=477.27 \text { units } \\ \text { 3. } & \text { Square } & : \text { Side }=1500 \text { units } \\ \text { 4. } & \text { Rectangle } & : \text { Length }=1875 \text { units Breadth }=1200 \text { units } \\ \text { 5. } & \text { Rectangle } & \text { : Length }=2400 \text { units Breadth }=937.50 \text { units } \\ \text { 6. } & \text { Rectangle } & : \text { Length }=3000 \text { units Breadth }=750 \text { units } \\ \text { 7. } & \text { Rectangle } & : \text { Length }=4800 \text { units Breadth }=468.75 \text { units }\end{array}$

\section{ACKNOWLEDGEMENTS}

The authors would like to thank everyone, just everyone!

\section{REFERENCES}

[1] Natrajan Meghnathan, "Impact of Range of Simulation Time and Network Shape on Hop Count and Stability of Routes in Mobile Ad-hoc Netwroks", IAENG Internationa Journal of Computer Science,36:1,IJCS_36_1_01

[2] Francois Ingelrest, David Simpol- Ryl, and Ivan Stojjmenovic, “ Optimal Transmission Radius for Energy Effiecinet Broadingcasting Protocol in Ad-hoc and Sensor Networks" IEEE Transactions on parallel and distributed systems, Vol. 17, No. 6, June 2006 
International journal on applications of graph theory in wireless ad hoc networks and sensor networks (GRAPH-HOC) Vol.3, No.3, September 2011

[3] Choong Hock MAR, Wnnston K.G.SEAH "An analysis of connectivity in a MANET of autonomous cooperative mobile agents under the Rayleigh fading channel", 0-7803-8887-9/05/\$20.00 ( c ) 2005 IEEE

[4] G. Ferrari and O. K. Tonguz. Minimum number of neighbours for fully connected uniform ad-hoc wireless networks. In IEEE Communication Society, volume 7, pages 4331- 4335, June 2004.

[5] Q. Ling and Z. Tian. Minimum node degree and k-connectivity of a wireless multihop network in bounded area. In GLOBECOM '07: Global Telecommunications Conference, pages 1296-1301. IEEE, November 2007

[6] L. Kleinrock and J. A. Silvester. Optimum transmission radii in packet radio networks or why six is a magic number. In Proceedings of IEEE National Telecommunications Conference, pages 04.3.104.3.5, December 1978.

[7] O. K. Tonguz and G. Ferrari. Is the number of neighbours in ad-hoc wireless networks a good indicator of connectivity? IEEE International Zurich Seminar on Communications : AccessTransmission-Networking, 18-20 Feb. 2004

[8] J. Wu and D. R. Stinson. Minimum node degree and k-connectivity for key predistribution schemes and distributed sensor networks. In WiSec '08: Proceedings of the first ACM conference on Wireless network security, pages 119-124. ACM, 2008.

[9] H. Takagi and L. Kleinrock. Optimal transmission ranges for randomly distributed packet radio terminals. IEEE Transactions on Communica-tions, 32:246-257, March 1984.

[10] T.-C. Hou and V. Li. Transmission range control in multihop packet radio networks. IEEE Transactions on Communications, 34:38-44, January 1986.

[11] S. Song, D. Goeckel, and D. F. Towsley. An improved lower bound to the number of neighbours required for the asymptotic connectivity of ad-hoc networks. IEEE Transactions on Information Theory, 2005.

[12] F.J. Ovalle-Martinez, I.Stojmenovic, F. Gracia-Nocetti and J. Solano-Gonzalez (2005) “ Finding minimum transmission radii for preserving connectivity and constructing spanning trees in ad-hoc sensor networks", Journal of Parallel and Disributed Computing, pp. 132-141.

[13] T. Camp, J. Boleng and V. Davies, "A Survey of mobility models for ad-hoc network research", Wireless Communications \& Mobile Computing (WCMC): Special issue on Mobile Ad-hoc Networking: Research, Trends and Applications, 2(5), 2002, pp 483-502.

[14] Guolong Lin, Guevara Noubir and Rajmohan Rajaraman, "Mobility Models for Ad hoc Network Simulation", In Proceedings of IEEE INFOCOM 2004, Volume 1, pp. 7-11, 2004.

[15] Tracy Camp, Jeff Boleng and Vanessa Davies, "A Survey of Mobility Models for Ad Hoc Network" Special issue on Mobile Ad Hoc Networking: Research, Trends and Applications, vol. 2, no. 5, pp. 483-502, 2002.

[16] F. Bai and A. Helmy, "The IMPORTANT Framework for Analyzing and Modeling the Impact of Mobility in Wireless Adhoc Networks", in Wireless Ad Hoc and Sensor Networks, Kluwer Academic Publishers, 2004. 
International journal on applications of graph theory in wireless ad hoc networks and sensor networks (GRAPH-HOC) Vol.3, No.3, September 2011

\section{Authors}

First Author: Chander Kumar is M.Tech (Computer Engg.) and Ph.D (Computer Science). His academic interests include Network security, software reliability and Artificial intelligence.

Dr. C.K.Nagpal

Associate Professor.(Computer Engg.)

YMCA University of Science and Technology,

Faridabad

E-mail: nagpalckumar@ rediffmail.com

Second Author: Shailender Gupta is B. Tech (Electronics) and M. Tech (Computer Engg.) and pursuing his $\mathrm{Ph}$. D in the area of ad-hoc mobile network security. His academic interests include network security, automata theory and fuzzy logic.

Shailender Gupta

Assistant Professor (Electronics Engg.)

YMCA University of Science and Technology,

Faridabad

E-mail: shailender81@gmail.com

Third Author: Bharat Bhushan is B. Tech (Electronics) and M.Tech (Electronics Engg.). His academic interests include Mobile Ad-hoc Network, Network Security.

Bharat Bhushan

Assistant Professor (Electronics Engg.)

YMCA University of Science and Technology,

Faridabad

E-mail: bhrts@yahoomail.com 